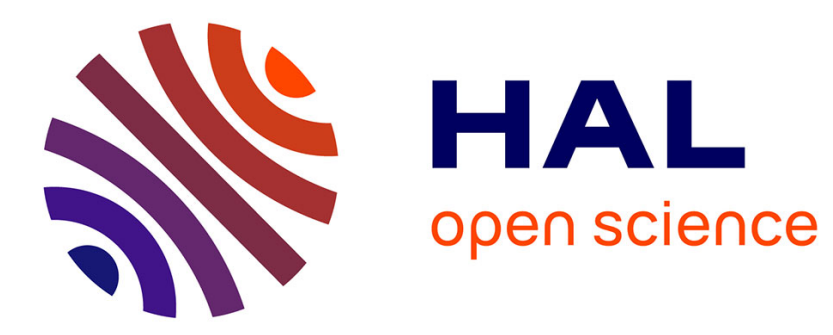

\title{
Discrete and continuum modelling of delamination processes
}

Czeslaw Wozniak

\section{To cite this version:}

Czeslaw Wozniak. Discrete and continuum modelling of delamination processes. Ingenieur-Archiv, 1990, 60 (5), pp.335-344. hal-00944248

\section{HAL Id: hal-00944248 \\ https://hal.science/hal-00944248}

Submitted on 18 Feb 2014

HAL is a multi-disciplinary open access archive for the deposit and dissemination of scientific research documents, whether they are published or not. The documents may come from teaching and research institutions in France or abroad, or from public or private research centers.
L'archive ouverte pluridisciplinaire HAL, est destinée au dépôt et à la diffusion de documents scientifiques de niveau recherche, publiés ou non, émanant des établissements d'enseignement et de recherche français ou étrangers, des laboratoires publics ou privés. 


\title{
Discrete and continuum modelling of delamination processes
}

\author{
Cz. Woźniak, Warszawa
}

Summary: The chaotic distributions of delaminated surface elements in laminates are described by a macrodelamination density function. Using this concept a phenomenological discrete mathematical model of the interlaminar debonding processes is proposed. For multilayered periodic composites the discrete model is approximated by a certain continuum model of delamination processes.

\section{Diskrete und kontinuierliche Modelle der Schichtentrennungsvorgänge}

Übersicht: Eine chaotische Verteilung von gelockerten Bereichen von den Trennflächen von Schichtwerkstoffen wird durch eine Dichtefunktion der Schichtentrennung modelliert. Diese Darstellung ermöglicht die Formulierung eines phänomenologischen diskreten Modells von Schichtentrennungsvorgängen. Für vielschichtige, periodische Verbundwerkstoffe wird das diskrete Modell durch ein kontinuierliches Modell approximiert.

\section{Introduetion}

In this paper we deal with composites made of a number of sheets (laminae) bonded together by very thin layers of a bonding material. We shall assume that the bonding layers can be treated as surfaces, i.e. they coincide with the interfaces of adjacent sheets; the scheme of the composite under consideration is shown in Fig. 1. We are to investigate deformation and stresses in such laminates taking into account the possibility of the debonding of laminae. The mathematical model of debonding processes in layered composites was proposed in [1] where the loss of a permanent contact between the sheets was uniquely determined by a certain extremal value of the strain energy of the bonding material. However, in many deformation processes met in the engineering practice a chaotic distribution of certain delaminated micro-surface elements between the adjacent layers can be observed. This chaotic distribution is due to the unknown a priori nonuniform and non-local character of bonding. Moreover, the model introduced in [1] is too involved to be the basis of engineering applications.

The main aim of this contribution is to propose and discuss two macroscopic (phenomenological) models of the debonding processes in laminates which take into account the chaotic micro-

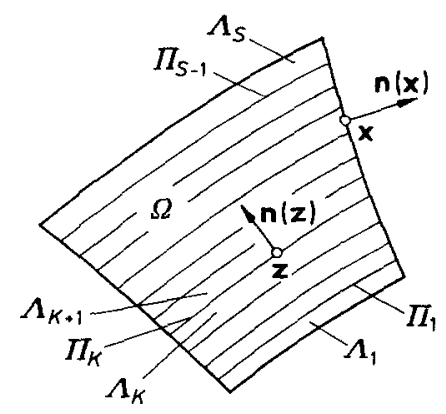

Fig. 1. Scheme of the composite 
delaminations between the interfaces of sheets. We start with the discrete macro-modelling of delamination described in terms of functions defined on the interlaminar surfaces; the leading concept here is that of a delamination density related to these surfaces. If the number of layers is big enough, then the discrete model is not plausible from the point of view of engineering applications. That is, we shall pass to the continuum model of debonding processes where the delamination density is related to the region occupied by the whole composite in its undeformed state. In the continuum modelling we apply the microlocal approach, [2], which takes into account some concepts of the nonstandard analysis, $[3,4]$. The proposed models are able to describe the physical situations met in engineering, in which only partial delamination in certain parts of a composite takes place.

The time-irreversibility of the debonding processes leads to time-nonlocal governing relations of the problems considered. However, for the quasi-stationary debonding processes it is shown that after time discretization we obtain a sequence of problems each of them being described by a certain variational inequality in a discrete model or a certain nonlinear equation in a continuum model. The solutions to these problems exist and are unique up to the time instant in which the total delamination takes place. The considerations are carried on under the following assumptions: $i$. The material of separate sheets is homogeneous and linear elastic,

$i$. the thin layers of the interlaminar bonding material are modelled as surfaces having linearelastic properties and able to transmit only restricted values of the interlaminar tractions,

iii. after the delamination only the unilateral contact without friction between the adjacent laminae is possible,

$i v$. the small deformation gradient theory can be applied.

\section{Micro- and macro-delamination}

Let $\Omega$ stands for the regular region in $\mathbb{R}^{3}$ occupied by the undeformed composite made of $S$ disjointed sheets $\Lambda_{K}(K=1, \ldots, S)$ separated by the interlaminar surfaces $\Pi_{K}(K=1, \ldots, S-1)$ as shown in Fig. 1.

Let the composite be subject to a certain deformation process in the time interval $\left[\tau_{0}, \tau_{f}\right]$ with the initial time instant $\tau_{0}$ related to the undeformed and unstressed body. Using the approach applied in [1] we assume that the displacement fields, defined for every $\tau \in\left[\tau_{0}, \tau_{f}\right]$ on $\Omega$, may suffer discontinuities across the interlaminar surfaces $\Pi_{K}$ and that some critical values of these discontinuities may lead to the loss of the permanent contact between the adjacent layers $A_{K}, A_{K+1}$. In this case the bonding material is no more able to transmit any interlaminar forces and the only interactions across the interfaces of sheets are due to the possible mutual unilateral contact between the sheets. Define

$$
\Lambda \equiv \bigcup_{K=1}^{S} \Lambda_{K}, \quad \Pi \equiv \bigcup_{K=1}^{S-1} \Pi_{K}
$$

The part of $\Pi$ where at the time instant $\tau \in\left[\tau_{0}, \tau_{f}\right]$ (in the process under consideration) such debonding takes place will be denoted by $D(\tau)$. From observations and experiments carried out with real laminates it follows that $D(\tau)$ consists of many chaotically distributed small surface elements. Hence $D(\tau)$ can be referred to as the micro-delaminated part of $I$ at the time instant $\tau$. The mapping

$$
\left[\tau_{0}, \tau_{f}\right] \ni \tau \rightarrow \chi_{D(\tau)}(\cdot), \quad \chi_{D(\tau)}(\mathbf{z}) \equiv\left\{\begin{array}{lll}
1 & \text { if } & \mathbf{z} \in D(\tau) \\
0 & \text { if } & \mathbf{z} \in I I \backslash D(\tau)
\end{array}\right.
$$

will be called the micro-delamination process. Obviously, $D\left(\tau_{1}\right) \subseteq D\left(\tau_{2}\right)$ for every $\tau_{1}<\tau_{2}$ due to the time irreversibility of this process.

In this contribution we are to propose a certain phenomenological model of the micro-delamination processes which will be the basis for the mathematical macro-modelling of debonding processes in multilayered composites. Let $h$ be the minimum thickness of the laminae and $B(z, r)$ 
be a ball in $\mathbb{R}^{3}$ with a center $\mathbf{z} \in I I$ and a radius $r$, such that $r<h$. Then

$$
\delta_{\tau}(\mathbf{z}, \tau) \equiv \frac{\operatorname{area}[B(\boldsymbol{z}, r) \cap D(\tau)]}{\operatorname{area}[B(\boldsymbol{z}, r) \cap \Pi]}
$$

represents the mean density of the micro-delamination at $z \in \Pi$ and at a time instant $\tau \in\left[\tau_{0}, \tau_{f}\right]$. The phenomenological model of delamination will be based on the heuristic assumption that in every debonding process there exist sufficiently regular functions $\delta(\cdot, \tau), \tau \in\left[\tau_{0}, \tau_{f}\right]$, defined almost everywhere on $I I$, such that $\delta(\cdot, \tau) \cong \delta_{r}(\cdot, \tau)$ for every $r \cong h$. The values $\delta(\mathbf{z}, \tau)$ will be called the delamination densities at $\mathbf{z} \in \Pi$ and at the time instant $\tau \in\left[\tau_{0}, \tau_{f}\right]$. Moreover, the mapping

$$
\left[\tau_{0}, \tau_{f}\right] \ni \tau \rightarrow \delta(\cdot, \tau), \quad \delta(\mathbf{z}, \tau) \in[0,1], \quad \mathrm{z} \in \Pi
$$

will be referred to as the macro-delamination process. It can be assumed that the micro- and macrodelamination processes are interrelated by

$$
\delta(\mathbf{z}, \tau)=\int_{\Pi_{k}} \hat{\varrho}(\mathbf{z}-\overline{\mathbf{z}}) \chi_{D(\tau)}(\overline{\mathbf{z}}) \mathrm{d} A(\overline{\mathbf{z}}), \quad \mathbf{z} \in \Pi_{K} \quad(K=1, \ldots, S-1)
$$

where $\hat{\varrho}(\cdot)$ is a certain function which makes it possible to treat the macro-delamination density $\delta(\cdot, \tau)$ as the result of a certain filtration of a chaotic distribution $D(\tau)$ of micro-delaminated parts of $\Pi$. The main feature of the macro-delamination process is that it takes into account what will be called the partial delamination states in which $0<\delta(\mathbf{z}, \tau)<1$ for some $\mathbf{z} \in \Pi$ and $\tau \in\left[\tau_{0}, \tau_{f}\right]$. The case $\delta(z, \tau)=1$ will be referred to as the total delamination at $\mathbf{z} \in \Pi$ and at the time instant $\tau$ of the deformation process; from the physical point of view this case can be interpreted as the micro-delamination in a certain vicinity of $\mathrm{z}$ on $I I$.

The heuristic concepts of the delamination density and that of the macro-delamination process constitute the basis for the macro-modelling of debonding processes in laminates. In the subsequent section the basic assumptions and governing relations for the discrete macro-model will be formulated.

In the sequel we denote by $\mathbf{n}(\mathbf{z}), \mathbf{z} \in \Pi$, the unit normal to $\Pi$ which for every $\mathbf{z} \in \Pi_{K}$ is outward to $A_{K}(K=1, \ldots, S-1)$. The unit outward normal to $\partial \Omega$ is denoted by $\mathbf{n}(\mathbf{x}), \mathbf{x} \in \partial \Omega$. For an arbitrary vector field $w: I I \rightarrow \mathbb{R}^{3}$ we introduce the notation

$$
w_{N}(\mathbf{z}) \equiv \mathbf{w}(\mathbf{z}) \cdot \mathbf{n}(\mathbf{z}), \quad \mathbf{w}_{T}(\mathbf{z}) \equiv \mathbf{w}(\mathbf{z})-\mathbf{n}(\mathbf{z}) w_{N}(\mathbf{z}) .
$$

Moreover, for every field $\psi(\cdot)$ defined on $A$, which has well defined traces on $\partial A$, we introduce the jump of this field across $\Pi$, setting

$$
\llbracket \psi \rrbracket(\mathbf{z}) \equiv \psi^{+}(\mathbf{z})-\psi^{-}(\mathbf{z}), \quad \mathbf{z} \in \Pi_{K} \quad(K=1, \ldots, S-\mathbf{1})
$$

where $\psi^{-}(\mathbf{z}), \psi^{-}(\mathbf{z})$ are the values of the pertinent traces on $\partial \Lambda_{K+1} \cap \Pi_{K}$ and $\partial A_{K} \cap \Pi_{K}$, respectjvely.

\section{Discrete macro-modelling}

Let for every time instant $\tau \in\left[\tau_{0}, \tau_{f}\right]$ the composite under consideration be subject to the known body forces $\mathbf{b}(\mathbf{x}, \tau), \mathrm{x} \in A$, and the surface tractions $\mathrm{p}(\mathbf{x}, \tau), \mathrm{x} \in \Gamma$, where $\Gamma$ is the corresponding part of the boundary $\partial \Omega$ of $\Omega$. The internal forces will be represented by the Cauchy stress tensor field $T(x, \tau), x \in A$, and the interlaminar stress vector field $t(z, \tau), z \in \Pi$. The displacement field at an arbitrary time instant $\tau \in\left[\tau_{0}, \tau_{f}\right]$ will be denoted by $\mathbf{u}(\mathbf{x}, \tau), \mathbf{x} \in A$. We assume that $\mathbf{u}(\cdot, \tau) \in V$ where $V$ is a linear topological space of sufficiently regular functions defined almost everywhere on $A$ which have well defined traces on $\partial A$, and may suffer discontinuities $\llbracket \mathbf{u} \rrbracket(\mathbf{z}, \tau), \mathbf{z} \in \Pi$, across the interlaminar surfaces $\Pi$. We also assume that on the part $\Gamma_{0}$ of the boundary $\partial \Omega$, the traces $\mathbf{u}_{0}(\mathbf{x}, \tau), \mathbf{x} \in \Gamma_{0}$, are known for every $\tau \in\left[\tau_{0}, \tau_{f}\right]$, and that mes $\left(\Gamma_{0}\right)>0$. In every problem under consideration the mass density $\varrho(\mathbf{x})$ and the tensor of elastic moduli $\mathbf{C}(\mathbf{x})$ are assumed to be known for almost every $\mathbf{x} \in A$. In the sequel we shall introduce the linear operator defined on $V$

$$
\mathscr{E} \mathbf{v} \equiv\left(\nabla \mathbf{v}+(\nabla \mathbf{v})^{T}\right) / 2
$$


and the space $V_{0}$ of the test functions

$$
V_{0}:=\left\{\mathbf{v} \in V:\left.\mathbf{v}\right|_{\Gamma_{0}}=\mathbf{0}\right\} .
$$

With forementioned denotations and assumptions introduced in Sec. 1 we obtain the stressstrain relations

$$
\mathbf{T}(\mathbf{x}, \tau)=\mathbf{C}(\mathbf{x})[\mathbf{E}(\mathbf{x}, \tau)], \quad \mathbf{E}(\mathbf{x}, \tau)=\mathscr{E} \mathbf{u}(\mathbf{x}, \tau) ; \quad \mathbf{x} \in A, \quad \tau \in\left[\tau_{0}, \tau_{f}\right]
$$

for the material of the laminae and the following variational form of the equations of motion:

$$
\begin{aligned}
& \int_{\Omega} \operatorname{tr}[\mathbf{T}(\mathbf{x}, \tau) \mathbf{E v}(\mathbf{x})] \mathrm{d} V+\int_{\Pi} \mathrm{t}(\mathbf{z}, \tau) \cdot \llbracket \mathbf{v} \rrbracket(\mathbf{z}) \mathrm{d} A=\int_{\Omega} \varrho(\mathbf{x})[\mathbf{b}(\mathbf{x}, \tau)-\ddot{\mathbf{u}}(\mathbf{x}, t)] \cdot \mathbf{v}(\mathbf{x}) \mathrm{d} V \\
& +\int_{\Gamma} \mathbf{p}(\mathbf{x}, \tau) \cdot \mathbf{v}(\mathbf{x}) \mathrm{d} A \quad \forall \mathbf{v}(\cdot) \in V_{0}, \quad \tau \in\left[\tau_{0}, \tau_{f}\right] .
\end{aligned}
$$

Using the approach described in [1] we also have

$$
\mathbf{t}(\mathbf{z}, \tau)=\mathbf{s}(\mathbf{z}, \tau)+\mathbf{n}(\mathbf{z}) r(\mathbf{z}, \tau), \quad \mathbf{z} \in \Pi, \quad \tau \in\left[\tau_{0}, \tau_{f}\right]
$$

where $\mathbf{s}(\mathbf{z}, \tau)$ represents the interlaminar stress due to the bonding material and $r(\mathbf{z}, \tau)$ is the value of the reaction to constraints $\left[\mathbf{u} \rrbracket_{N}(\mathbf{z}, \tau) \geqq 0, \mathbf{z} \in \Pi\right.$, resulting from the impenetrability of adjacent laminae. Introducing the cone $\mathbb{K}$ in $V_{0}$ defined by

$$
\mathbb{K}:=\left\{\mathbf{v} \in V_{0}: \llbracket \mathbf{v} \rrbracket_{N}(\mathbf{z}) \geqq 0 \text { for almost every } \mathbf{z} \in \Pi\right\}
$$

we obtain

$$
\begin{aligned}
& \mathbf{u}(\cdot, \tau) \in \mathbb{K}, \\
& \int_{I}(\llbracket \mathbf{v} \rrbracket(\mathbf{z})-\llbracket \mathbf{u} \rrbracket(\mathbf{z}, \tau)) \cdot \mathbf{n}(\mathbf{z}) r(\mathbf{z}, \tau) \mathrm{d} A \geqq 0, \quad \forall \mathbf{v} \in \mathbb{K}, \quad \tau \in\left[\tau_{0}, \tau_{f}\right] .
\end{aligned}
$$

So far, the approach coincides with that given in [1]. The crucial point of the modelling is to propose an interrelation between the interlaminar bonding stresses $\mathbf{s}(\mathbf{z}, \tau)$, the delamination density $\delta(\mathbf{z}, \tau)$ and the displacement jumps $\llbracket \mathbf{u} \rrbracket(\mathbf{z}, \tau), \mathbf{z} \in \Pi$, in the debonding process. To this aid we introduce the two following real valued functions:

1. The interlaminar strain energy function $\pi(\mathbf{v}), \mathbf{v} \in \mathbb{R}^{3}$, which describes the linear elastic properties of the bonding material before possible macro-delamination (i.e. for $\delta(z, \tau)=0$ ):

$$
\pi(\mathbf{v})=\frac{1}{2}\left(\gamma_{N}\left|v_{N}\right|^{2}+\gamma_{T}\left|\mathbf{v}_{T}\right|^{2}\right),
$$

where $\gamma_{N}, \gamma_{T}$ are the longitudinal and shear moduli, respectively. Introducing for every $\mathbf{v} \in \mathbb{R}^{3}$ the norm

$$
|\mathbf{v}|_{\xi} \equiv \sqrt{\left(\mathbf{v}_{T}\right)^{2}+\xi\left(v_{N}\right)^{2}}, \quad \xi>0,
$$

and setting $\xi=\gamma_{N} / \gamma_{T}, \mathbf{v}=\llbracket \mathbf{u} \rrbracket(\mathbf{z}, \tau)$, we obtain

$$
\pi(\llbracket \mathbf{u} \rrbracket(\mathbf{z}, \tau))=\frac{1}{2} \gamma_{T}|\llbracket \mathbf{u} \rrbracket(\mathbf{z}, \tau)|_{\xi}^{2} .
$$

Using the denotations $\varepsilon(\mathbf{z}, \tau) \equiv|\llbracket \mathbf{u} \rrbracket(\mathbf{z}, \tau)|_{\xi}, \sigma(\mathbf{z}, \tau) \equiv \pi(\llbracket \mathbf{u} \rrbracket(\mathbf{z}, \tau))$ we also get $\sigma(\mathbf{z}, \tau)=0.5 \gamma_{T} \varepsilon^{2}(\mathbf{z}, \tau)$. 2. The delamination density function $\delta_{E}(\sigma), \sigma \geqq 0$, which determines the delamination density $\delta(\mathbf{z}, \tau)$ in term of the supremum of the interlaminar strain energy at $\mathbf{z} \in \Pi$ in the time interval $\left[\tau_{0}, \tau\right)$. We assume that $\delta_{E}(\cdot)$ is a continuous monotone function such that for some $\sigma_{0}, \sigma_{1}\left(0 \leqq \sigma_{0}<\sigma_{1}\right)$ we have $\delta_{E}(\sigma)=0$ if $\sigma \in\left[0, \sigma_{0}\right]$, and $\delta_{E}(\sigma)=1$ if $\sigma \in\left[\sigma_{1}, \infty\right)$. In the simplest case we can assume that $\delta_{E}(\cdot)$ is linear in $\left[\sigma_{0}, \sigma_{1}\right]$. form

Setting $\mathbf{S v} \equiv \partial \pi(\mathbf{v}) / \partial \mathbf{v}, \mathbf{v} \in \mathbb{R}^{3}$, the interlaminar bonding relations will be postulated in the

$$
\mathbf{s}(\mathbf{z}, \tau)=[1-\delta(\mathbf{z}, \tau)] \mathbf{S} \llbracket \mathbf{u} \rrbracket(\mathbf{z}, \tau), \quad \mathbf{z} \in \Pi, \quad \tau \in\left[\tau_{0}, \tau_{f}\right]
$$


where

$$
\delta(\mathbf{z}, \tau)=\delta_{E}\left(\sup _{\overline{\mathbf{z}} \in \mathbf{i} \mathbf{0}, \tau)} \pi(\llbracket \mathbf{u} \rrbracket(\mathbf{z}, \bar{\tau})), \quad \mathbf{z} \in \Pi, \quad \tau \in\left[\tau_{0}, \tau_{f}\right]\right.
$$

It can be seen that the functional in (3.6) describes the time irreversibility of the debonding process. Alternatively, setting $\delta_{D}(\varepsilon) \equiv \delta_{E}\left(0.5 \gamma_{T} \varepsilon^{3}\right), \varepsilon \geqq 0$, we can replace (3.6) by

$$
\delta(\mathbf{z}, \tau)=\delta_{D}\left(\sup _{\bar{\tau} \in\left[\tau_{0}, \tau\right)}|\mathbb{[ u} \rrbracket(\mathbf{z}, \bar{\tau})|_{\xi}\right), \quad \mathbf{z} \in M, \quad \tau \in\left[\tau_{0}, \tau_{f}\right] .
$$

We see that if the delamination density increases, then the bonding material is able to transmit smaller values of the interlaminar tractions $\mathbf{s}(\mathbf{z}, \tau)$.

Together with the initial conditions

$$
\mathbf{u}\left(\mathbf{x}, \tau_{0}\right)=\mathbf{0}, \quad \dot{\mathbf{u}}\left(\mathbf{x}, \tau_{0}\right)=\mathbf{v}_{0}(\mathbf{x}), \quad \mathbf{x} \in \Omega,
$$

(3.1) - (3.5) and the conditions (3.6) or (3.7) constitute the governing relations of the discrete model for delamination processes in the layered composites under consideration. The term discrete is used to underline the fact that the possible debonding takes place on the discret set of interlaminar surfaces $I_{K}(K=1, \ldots, S-1)$.

\section{Analysis of diserete models}

For the sake of simplicity we confine ourselves to the quasi-stationary debonding processes. Moreover, applying the time-local approximation introduced in [1] we shall assume that the governing relations (3.1) $-(3.5)$ have to hold only for $\tau=\tau_{A}(A=0,1, \ldots, M)$ where $\tau_{0}, \tau_{1}, \ldots, \tau_{M} \equiv \tau_{f}$ constitute the finite sequence of time instants such that $\tau_{0}<\tau_{1}<\cdots<\tau_{M}$. Then for every fixed $\tau_{A}$ the values of $\delta\left(\mathbf{z}, \tau_{A}\right), z \in \Pi$, in (3.5) are known, provided that the solutions to the problems described by $(3.1)-(3.6)$ have been succesively calculated for $\tau_{1}^{\prime}, \tau_{2}, \ldots, \tau_{A-1}$, cf. [1]. Every such problem, from the formal point of view, can be treated as time-independent. Setting aside the argument $\tau$ and introducing the denotations

$$
\begin{aligned}
& a_{\delta}(\mathbf{u}, \mathbf{v}) \equiv \int_{A} \operatorname{tr}[\mathscr{E} \mathbf{v}(\mathbf{x})(\mathbf{C}[\mathscr{E} \mathbf{u}](\mathbf{x}))] \mathrm{d} V+\int_{\Pi \backslash(\delta)}[1-\delta(\mathbf{z})] \llbracket \mathbf{v} \rrbracket(\mathbf{z}) \cdot \mathbf{s} \llbracket \mathbf{u} \rrbracket(\mathbf{z}) \mathrm{d} A, \\
& \Delta(\delta):=\{\mathbf{z} \in \Pi: \delta(\mathbf{z})=\mathbf{1}\} \\
& \langle\mathbf{v}, f\rangle \equiv \int_{\Lambda} \varrho(\mathbf{x}) \mathbf{b}(\mathbf{x}) \cdot \mathbf{v}(\mathbf{x}) \mathrm{d} V+\int_{\Gamma} \mathbf{p}(\mathbf{x}) \cdot \mathbf{v}(\mathbf{x}) \mathrm{d} A
\end{aligned}
$$

we obtain from (3.1)-(3.5) the following variational inequality

$$
a_{\delta}(\mathbf{v}-\mathbf{u}, \mathbf{u}) \geqq\langle\mathbf{v}-\mathbf{u}, f\rangle \forall \mathbf{v} \in \mathbb{K}, \quad \mathbf{u} \in \mathbb{K},
$$

the form of which depends on the previously calculated delamination density $\delta(\cdot)$.

Using the known denotation for the Sobolev spaces define

$$
V:=\left\{\mathrm{v} \in\left(L^{2}(\Omega)\right)^{3}:\left.\mathrm{v}\right|_{A K} \in\left(H^{1}\left(\Lambda_{K}\right)\right)^{3} \quad(K=1, \ldots, S)\right\}
$$

and let $V^{*}$ be a dual of $V$. Then under the condition that mes $\left(\Pi_{K} \backslash \Delta(\delta)\right)>0(K=1, \ldots, S-1)$ it can be proved that for every $f \in V^{*}$ and every $\Gamma_{0}$ such that mes $\left(\Gamma_{0}\right)>0$, there exist the unique solution $\mathbf{u}=\mathbf{u}(\cdot)$ to the variational inequality (4.1). The exact analysis of this problem will be detailed separately.

Summarizing the obtained result we conclude that the quasi-stationary debonding processes in the laminates under consideration can be described by the sequence of variational inequalities for the displacement fields $\mathbf{u}\left(\tau_{A}\right) \equiv \mathbf{u}\left(\cdot, \tau_{A}\right),(A=1,2, \ldots, M)$ given by

$$
a_{\delta\left(\tau_{A}\right)}\left(\mathbf{v}-\mathbf{u}\left(\tau_{A}\right), \mathbf{u}\left(\tau_{A}\right)\right) \geqq\left\langle\mathbf{v}-\mathbf{u}\left(\tau_{A}\right), \quad f\left(\tau_{A}\right)\right\rangle \forall \mathbf{v} \in \mathbb{K}, \quad \mathbf{u}\left(\tau_{A}\right) \in \mathbb{K}
$$


where the delamination density $\delta(\tau)=\delta(\cdot, \tau)$ is determined by

$$
\delta\left(\mathbf{z}, \tau_{A}\right)=\delta_{D}\left(\sup _{B \in\{1, \ldots, A-1\}}\left|\llbracket \mathbf{u} \rrbracket\left(\mathbf{z}, \tau_{B}\right)\right|_{\xi}\right), \quad \mathbf{z} \in \Pi .
$$

The problems described by (4.2) can be succesively solved provided that the totally delaminated part $\Delta\left(\delta\left(\tau_{A}\right)\right)$ of the interlaminar surfaces $I$ satisfies the condition in the above stated theorem.

The time-local approximation can be also applied to dynamical delamination processes. In this case we arrive at the sequence of dynamical problems for functions $\left[\tau_{A}, \tau_{A+1}\right] \ni \tau \rightarrow \mathbf{u}(\tau) \in V$, satisfying the time dependent variational inequalities and the pertinent initial conditions.

\section{$5 \quad$ Continuum modelling}

In many engineering problems we deal with laminates made of a large numer of repeating basic units of laminae, every unit being composed of a few sheets. Such composites have a periodic structure in the direction $\mathbf{n}(\mathbf{z}), \mathbf{z} \in \Pi_{K}(K=1, \ldots, S-1)$. Due to the large number of interfaces $\Pi_{K}$, across which the displacement field can suffer a discontinuity, the discrete models of delamination processes, introduced in Sec. 3 , are not plausible in the engineering applications of the theory (e.g. as a basis of numerical calculations). That is why we shall pass now to the continuum models of macro-delamination processes. The approach outlined below is based on the microlocal homogenization approach, [2].

Let every basic unit of the multilayered composite have the thickness $\varepsilon$ (in the undeformed state) and made of $s$ thin homogeneous sheets. Let $\mathbf{C}_{a}, \varrho_{a}(a=1, \ldots, s)$ stand for the tensors of elastic moduli and the mass densities, resp., in the particular sheets belonging to an arbitrary basic unit of the laminate. The fragment of the basic unit is shown on Fig. 2 where also the local coordinates $\zeta=\zeta(\mathbf{x}), \zeta \in[0, \varepsilon]$, is indicated.

Let $\mathbf{P}^{1}$ stand for the problem that can be stated as follows: for the periodic laminate with the basic layer of thickness $\varepsilon$ ( $\varepsilon$-periodic laminate) find displacements $\mathbf{u}(\cdot, \tau)$ and stresses $\mathbf{T}(\cdot, \tau)$, $\tau \in\left[\tau_{0}^{\prime}, \tau_{f}\right]$, satisfying (3.1)-(3.8) under pertinent regularity conditions, provided that the material structure and all external agencies acting on the body are known.

Let $\mathbf{P}^{n}(n=2,3, \ldots)$ stand for problems obtained from $\mathbf{P}^{1}$ by formal replacing the $\varepsilon$-periodic structure by $\varepsilon / n$-periodic structure, all remaining entities in (3.1)-(3.8) being unchanged. Roughly speaking, we pass from the periodic composite under consideration to a certain periodic composite in which the basic units of laminae are $n$-times thinner, but all other quantities in the problem under consideration remain unchanged. The method of modelling proposed in [2] and applied below is based on the following.

Homogenization assumption. The solution to the problem $\mathbf{P}^{\mathbf{1}}$ can be approximated for every $n=2$, $3,4, \ldots$ by the solution to the problem $\mathbf{P}^{n}$.

The forementioned assumption implies that the periodic laminate under consideration has sufficiently thin basic units (related to all characteristic length dimensions) and hence its macromaterial properties can be approximately treated as independent of the parameter $\varepsilon$.

The microlocal approach we are to apply, [2], is based on some notions and theorems of the

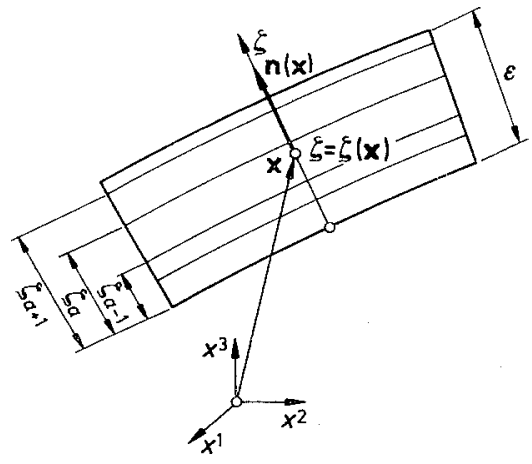

Fig. 2. Scheme of the basic unit in a periodic composite $\left(\zeta_{0}=0, \zeta_{s}=\varepsilon, \zeta \in[0, \varepsilon]\right)$ 
nonstandard analysis, $[3,4]$. In the nonstandard analysis we deal not only with the well known standard real numbers but also with the real numbers that are infinitely large and infinitely small. From the transfer principle of the nonstandard analysis it follows that if the problem $\mathscr{P} 1$ can be approximated by the problem $\mathscr{P}^{n}$ for every positive integer $n$, then it can be also approximated by the nonstandard problem $\mathscr{P}^{\omega}$ where $\omega$ is a certain infinite positive integer, i.e. a natural number that is greater then all standard natural numbers. From a formal point of view, $\mathcal{P}^{\omega}$ is a problem of a composite made of an infinite number of infinitely thin basic units having the thickness $\varepsilon / \omega$. It has to be emphasized that no limit passage is applied here and that the problem $\mathscr{P}^{n}$, $\mathfrak{P}^{w}$ have only formal meaning as certain approximations of the problem $\mathfrak{P}^{1}$.

In order to formulate the second assumption of the microlocal method of modelling, we introduce the functions $h_{a}: \mathbb{R} \rightarrow \mathbb{R}(a=1, \ldots, s-1)$ and $i_{A}: \mathbb{R} \rightarrow \mathbb{R}(A=1, \ldots, s)$ which are $\varepsilon$ periodic and given by

$$
\begin{aligned}
& h_{a}(\zeta)=\left\{\begin{array}{ccc}
0 & \text { if } & \zeta \in\left[0, \zeta_{a-1}\right] \cup\left[\zeta_{a+1}, \varepsilon\right], \\
\left(\zeta-\zeta_{a-1}\right) / \eta_{a-1} & \text { if } & \zeta \in\left[\zeta_{a-1}, \zeta_{a}\right], \\
\left(\zeta_{a+1}-\zeta\right) / \eta_{a} & \text { if } & \zeta \in\left[\zeta_{a}, \zeta_{a+1}\right],
\end{array}\right. \\
& i_{A}(\zeta)=\left\{\begin{array}{lll}
-\zeta+\zeta_{a}-\varepsilon / 2 & \text { if } \quad \zeta \in\left[0, \zeta_{a}\right), \\
-\zeta+\zeta_{a}+\varepsilon / 2 & \text { if } & \zeta \in\left(\zeta_{a}, \varepsilon\right]
\end{array}\right.
\end{aligned}
$$

where $\eta_{a} \equiv\left(\zeta_{a+1}-\zeta_{a}\right) / \varepsilon$ and $\zeta_{a}$ is shown in Fig. 2. The main idea of the microlocal modelling is to look for the solution to the problem $\mathscr{g}^{w}$ in a certain class of functions which involves only standard functions as the basic unknowns. For the problem under consideration we shall introduce the Microlocal approximation assumption. The displacement fields $\mathbf{u}(\cdot, \tau), \tau \in\left[\tau_{0}, \tau_{f}\right]$, representing a solution to the problem $\mathscr{g}^{\omega}$ can be approximated by $\tilde{\mathbf{u}}(\cdot, \tau)$, such that

$$
\tilde{\mathbf{u}}(\mathbf{x}, \tau)=*_{\mathbf{W}}(\mathbf{x}, \tau)+\frac{1}{\omega} * h_{\sigma}(\omega \zeta)^{*} q^{a}(\mathbf{x}, \tau)+\frac{1}{\omega} *_{i_{A}}(\omega \zeta)^{*} \mathbf{d}^{A}(\mathbf{x}, \tau), \quad \zeta=\zeta(\mathbf{x}), \quad \mathbf{x} \in *^{*} \Omega
$$

where the summation convention with respect to $a=1, \ldots, s-1$ and $A=1, \ldots, s$ holds, and where ${ }^{*} q^{a}(\cdot),{ }^{*} \mathbf{d}^{A}(\cdot),{ }^{*} \mathbf{W}(\cdot)$ are standard sufficiently regular unknown functions.

Using the known extension principle of the nonstandard analysis, cf. [3], we assign to every mathematical entity $F$ the entity * $F$ that is called standard. It means that the standard functions ${ }^{*} q^{a}(\cdot),{ }^{*} \mathbf{d}^{A}(\cdot),{ }^{*} \mathbf{W}(\cdot)$ are uniquely determined by certain functions $\mathbf{q}^{\alpha}(\cdot), \mathbf{d}^{A}(\cdot), \mathbf{w}(\cdot)$, resp., of the well known standard analysis. Functions $\mathbf{w}(\cdot, \tau), \tau \in\left[\tau_{0}, \tau_{f}\right]$, are called the macro-displacement fields and approximate the displacements of the composite at the time instant $\tau$. It can be observed that the terms in the microlocal approximation assumption involving $\mathbf{q}(\cdot), \mathbf{d}^{A}(\cdot)$ are infinitely small and can be neglected if we restrict ourselves only to the evaluation of displacements. However, in the evaluation of the displacement gradients all terms in the microlocal approximation assumption are involved. The functions $\mathbf{q}^{a}(\cdot, \tau)(a=1, \ldots, s-1)$ are referred to as microlocal parameters; it can be shown that they describe the effects due to the jumps of material properties between the adjacent layers, [2]. The functions $\mathrm{d}^{A}(\cdot, \tau)$ describe the jumps of displacements across the interfaces of the periodic laminate; we have to notice that the known functions $i_{A}(\cdot)$ are discontinuous. Setting

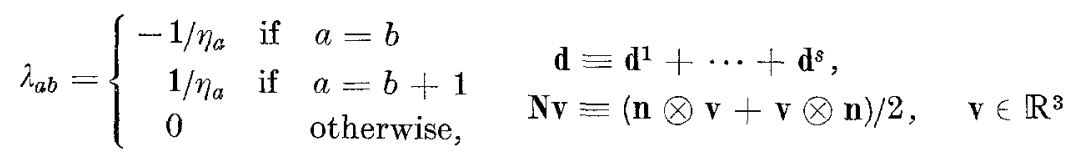

we can see that the strain tensors $\mathbf{E}_{G}\left(\mathbf{x}, \tau^{\prime}\right)$ in the $a$-th material component are determined by

$$
\mathbf{E}_{a}(\mathbf{x}, \tau)=\mathscr{E} \mathbf{w}(\mathbf{x}, \tau)+\lambda_{c b} \mathbf{N} \mathbf{q}^{b}(\mathbf{x}, \tau)-\mathbf{N} \mathbf{d}(\mathbf{x}, \tau) \quad(a=1, \ldots, s-1) .
$$

The vector fields $\mathbf{d}(\cdot, \tau), \tau \in\left[\tau, \tau_{f}\right]$, will be referred to as the strain incompatibilities.

The main feature of the microlocal modelling is that the non-standard problem $\mathscr{P}^{\omega}$ under the constraints for displacements given by the microlocal approximation assumption implies a certain problem $\widetilde{\mathscr{P}}$ for the macro-displacements $\mathbf{w}(\cdot, \tau)$, microlocal parameters $\mathbf{q}^{a}(\cdot, \tau)$ and strain 
incompatibilities $\mathbf{d}(\cdot, \tau), \tau \in\left[\tau_{0}, \tau_{f}\right]$. At the same time the problem $\tilde{\mathbf{P}}$ does not involve any nonstandard entity and represents a certain homogenized model of the $\varepsilon$-periodic laminate under consideration.

Neglecting here all calculations leading from the problem $\mathscr{P}^{\omega}$ to the problem $\tilde{\mathcal{P}}$ (for the particulars cf. [2]) we present below only the governing relations of the problem $\tilde{\mathscr{P}}$. They comprize:

1. The equations of motion

$$
\begin{aligned}
& \int_{\Omega} \operatorname{tr}[\tilde{\mathbf{T}}(\mathbf{x}, \tau) \mathscr{E} \mathbf{v}(\mathbf{x})] \mathrm{d} V=\int_{\Omega} \tilde{\varrho}[b(\mathbf{x}, \tau)-\ddot{\mathbf{w}}(\mathbf{x}, \tau)] \cdot \mathbf{v}(\mathbf{x}) \mathrm{d} V+\int_{\Gamma} \mathbf{p}(\mathbf{x}, \tau) \cdot \mathbf{v}(\mathbf{x}) \mathrm{d} A \\
& \forall \mathbf{v} \in V_{0}, \quad \mathbf{w}(\cdot, \tau) \in V_{0}, \quad \tau \in\left[\tau_{0}, \tau_{f}\right]
\end{aligned}
$$

where $\tilde{\mathbf{T}}(\mathbf{x}, \tau)$ is called the mean stress tensor, $\varrho$ is the mean mass density defined by $\varrho \cong \equiv \eta_{1} \varrho_{1}$ $+\cdots+\eta_{s} \varrho_{s}$ and the functional space $V_{0}$ coincides with that introduced in Sec. 3.

2. The constitutive equations for the mean stress

$$
\mathbf{T}_{a}(\mathbf{x}, \tau)=\mathbf{C}_{c}\left[\mathbf{E}_{a}(\mathbf{x}, \tau)\right], \quad \widetilde{\mathbf{T}}(\mathbf{x}, \tau)=\sum_{a=1}^{s} \eta_{a} \mathbf{T}_{a}(\mathbf{x}, \tau), \quad \mathbf{x} \in \Omega, \quad \tau \in\left[\tau_{0}, \tau_{f}\right]
$$

where $\mathbf{E}_{a}(\mathbf{x}, \tau)$ is given by (5.1).

3. The stress continuity conditions

$$
\left[\mathbf{T}_{a}(\mathbf{x}, \tau)-\mathbf{T}_{a+1}(\mathbf{x}, \tau)\right] \mathbf{n}(\mathbf{x})=\mathbf{0} \quad(a=1, \ldots, s-\mathbf{1}), \quad \mathbf{x} \in \Omega, \quad \tau \in\left[\tau_{0}, \tau_{f}\right]
$$

where $\mathbf{n}(\mathbf{x}), \mathbf{x} \in \Omega$, are unit vectors directed along the local coordinate $\zeta=\zeta(\mathbf{x})$ and the constitutive stress $\mathbf{T}_{a}(\mathbf{x}, \tau)$ is determined by means of $(5.3)$.

4. The averaged interlaminar conditions

$$
\begin{array}{ll}
\tilde{\mathbf{T}}(\mathbf{x}, \tau) \mathbf{n}(\mathbf{x})=\mathbf{s}(\mathbf{x}, \tau)+\mathbf{n}(\mathbf{x}) r(\mathbf{x}, \tau), & \\
\int_{\Omega}[\mathbf{u}(\mathbf{x})-\mathbf{d}(\mathbf{x}, \tau)] \cdot \mathbf{n}(\mathbf{x}) r(\mathbf{x}, \tau) \mathrm{d} V \geqq 0, \quad \forall \mathbf{u} \in \mathbb{K}_{n}, \mathbf{d}(\cdot, \tau) \in \mathbb{K}_{n}, \\
\mathbf{s}(\mathbf{x}, \tau)=[1-\delta(\mathbf{x}, \tau)] \mathbf{S} \mathbf{d}(\mathbf{x}, \tau), \\
\delta(\mathbf{x}, \tau)=\delta_{D}\left(\sup _{\bar{\tau} \in\left[\tau_{0}, \tau\right)}|\mathbf{d}(\mathbf{x}, \bar{\tau})|_{\xi}\right) ; \quad \mathbf{x} \in \Omega, & \tau \in\left[\tau_{0}, \tau_{f}\right]
\end{array}
$$

where the linear operator $\mathbf{S}$ and the density delamination function were introduced in Sec. 3 and where $\mathbb{K}_{n}$ is a cone in a certain linear topological space $W$ of vector functions defined almost everywhere on $\Omega$ by $\mathbb{K}_{n}:=\{\mathbf{u} \in W: \mathbf{u}(\mathbf{x}) \cdot \mathbf{n}(\mathbf{x}) \geqq 0, \mathbf{x} \in \Omega\}$.

5. The initial conditions

$$
\mathbf{w}\left(\mathbf{x}, \tau_{0}\right)=\mathbf{0}, \quad \dot{\mathbf{w}}\left(\mathbf{x}, \tau_{0}\right)=\mathbf{v}_{0}(\mathbf{x}), \quad \mathbf{x} \in \Omega
$$

where $\mathbf{v}_{0}(\cdot)$ is the known field belonging to $V_{0}$.

Under the pertinent regularity conditions $(5.1)-(5.6)$ represent the continuum model for the delamination processes in the periodic multilayered composites. The term continuum model is used in order to emphasize the fact that the delamination density $\delta(\mathbf{x}, \tau)$ is related here to the region $Q$ of $\mathbb{R}^{3}$ occupied by the laminate in its undeformed state. Hence the partial as well as total debonding has to be understood in the averaged form and can take place on certain subsets of $\Omega$.

\section{Analysis of continuum models}

It can be proved that the microlocal parameters $q^{\alpha}(x, \tau)$ together with the constitutive strains $\mathbf{E}_{a}(\mathbf{x}, \tau)$ and constitutive stresses can be eliminated from the governing relations listed above. From (5.1), (5.3) and (5.4) we obtain

$$
\widetilde{\mathbf{T}}(\mathbf{x}, \tau)=\widetilde{\mathbf{C}}[\mathscr{E} \mathbf{W}(\mathbf{x}, \tau)-\mathbf{N d}(\mathbf{x}, \tau)], \quad \mathbf{x} \in \Omega, \quad \tau \in\left[\tau_{0}, \tau_{f}\right]
$$


where $\tilde{\mathbf{C}}$ is the constant effective tensor of elastic moduli (for the particulars cf. [2]). At the same time (5.5) yields

$$
\begin{aligned}
& (\widetilde{\mathbf{T}}(\mathbf{x}, \tau) \mathbf{n}(\mathbf{x}))_{N} \in \partial \operatorname{ind}_{[0, \infty]}\left(d_{N}(\mathbf{x}, \tau)\right)+[1-\delta(\mathbf{x}, \tau)] \gamma_{N} d_{N}(\mathbf{x}, \tau), \\
& (\widetilde{\mathbf{T}}(\mathbf{x}, \tau) \mathbf{n}(\mathbf{x}))_{T}=[1-\delta(\mathbf{x}, \tau)] \gamma_{T} d_{T}(\mathbf{x}, \tau) ; \quad \mathbf{x} \in \Omega, \quad \tau \in\left[\tau_{0}, \tau_{f}\right]
\end{aligned}
$$

where we have applied the known denotation $\partial$ ind $_{[0, \infty]}$ for the subdifferential of the indicator function of $[0, \infty]$ and where we have denoted

$$
(\mathbf{v}(\mathbf{x}))_{N} \equiv \mathbf{n}(\mathbf{x}) \cdot \mathbf{v}(\mathbf{x}), \quad(\mathbf{v}(\mathbf{x}))_{T} \equiv \mathbf{v}(\mathbf{x})-\mathbf{n}(\mathbf{x})(\mathbf{v}(\mathbf{x}))_{N}, \quad \mathbf{v}(\mathbf{x}) \in \mathbb{R}^{3},
$$

for an arbitrary vector field $\mathrm{v}(\cdot)$ defined on $\Omega$. The crucial point of our procedure is to show that (6.1) and (6.2) imply

$$
\widetilde{T}(\mathbf{x}, \tau)=\mathbf{G}_{\delta(x, \tau)}(\mathscr{E} \mathbf{w}(\mathbf{x}, \tau)) ; \quad \mathbf{x} \in \Omega, \quad \tau \in\left[\tau_{0}, \tau_{f}\right],
$$

where $G_{\delta(x, t)}(\cdot)$ is the monotone nonlinear operator defined on the set of all symmetric $3 \times 3$ matrices. Moreover, if $\delta(\mathbf{x}, \tau)<1$, then $\mathbf{G}_{\delta(x, \tau)}(\cdot)$ is strongly monotone. In most cases the effective moduli tensor $\tilde{\mathbf{C}}$ related to the orthonormal vector basis $\mathbf{e}_{i}(\mathbf{x})$ with $\mathbf{e}_{3}(\mathbf{x})=\mathbf{n}(\mathbf{x})$ has a plane of elastic symmetry normal to $\mathbf{n}(\mathbf{x})$. With the denotation $\widetilde{\mathbf{C}}^{i j}[\mathbf{E}] \equiv \widetilde{C}^{i j k l} E_{k l}$ (all indices run over $1,2,3$, summation convention holds) and assuming that $\alpha, \beta$ run over 1,2 , we obtain the following explicit form of the mapping (6.3):

$$
\begin{aligned}
\tilde{T}^{\alpha \beta} & =\tilde{\mathbf{C}}^{\alpha \beta}[\mathscr{E} \mathbf{W}]-\frac{\tilde{C}^{\alpha \beta 33}}{\tilde{C}^{3333}+(1-\delta) \gamma_{N}}\left(\tilde{\mathbf{C}}^{33}[\mathscr{E} \mathbf{W}]\right)^{+}, \\
\tilde{T}^{33} & =\widetilde{\mathbf{C}}^{33}[\mathscr{E} \mathbf{W}]-\frac{\tilde{C}^{3333}}{\tilde{C}^{3333}+(1-\delta) \gamma_{N}}\left(\tilde{\mathbf{C}}^{33}[\mathscr{E} \mathbf{w}]\right)^{+}, \\
\tilde{T}^{\alpha 3} & =A_{. \beta}^{\alpha}(\delta) \tilde{\mathbf{C}}^{\beta 3}[\mathscr{E} \mathbf{W}],
\end{aligned}
$$

where $(\alpha)^{+} \equiv \max \{0, \alpha\}$ and where $A_{. \beta}^{\alpha}(\delta)$ are elements of the $2 \times 2$-matrix which is the inverse

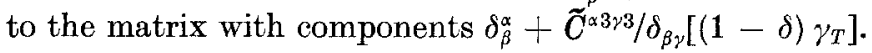

Summing up, we arrive at the conclusion that the continuum model of macro-delamination processes in multilayered periodic laminates is governed by the variational equation (5.2), the nonlinear monotone constitutive equation (6.3), the constitutive relation for the delamination density

$$
\delta(\mathbf{x}, \tau)=\delta_{D}\left(\sup _{\bar{\tau} \in\left\{\mathbf{T}_{0}, \tau\right)}|\mathbf{d}(\mathbf{x}, \bar{\tau})|_{\xi}\right), \quad \mathbf{d}(\mathbf{x}, \bar{\tau})=\frac{\mathbf{n}(\mathbf{x})[\widetilde{\mathbf{T}}(\mathbf{x}, \bar{\tau}) \mathbf{n}(\mathbf{x})]_{N}^{+}}{[1-\delta(\mathbf{x}, \bar{\tau})] \gamma_{N}}+\frac{\left(\left.\widetilde{\mathbf{T}}(\mathbf{x}, \bar{\tau}) \mathbf{n}(\mathbf{x})\right|_{T}\right.}{[1-\delta(\mathbf{x}, \bar{\tau})]_{T}},
$$

and by the initial conditions (5.6).

For the sake of simplicity let us confine ourselves to the quasi-stationary problems. Let us also apply the time-local approximation, following the approach described in Sec. 4. With the denotations

$$
\begin{aligned}
& (\mathscr{E} \mathbf{v}, \tilde{\mathbf{T}}) \equiv \int_{\Omega} \operatorname{tr}[\tilde{\mathbf{T}}(\mathbf{x}) \mathscr{E} \mathbf{v}(\mathbf{x})] \mathrm{d} V, \\
& \langle\mathbf{v}, f\rangle \equiv \int_{\Omega} \tilde{\varrho} b(\mathbf{x}) \cdot \mathbf{v}(\mathbf{x}) \mathrm{d} V+\int_{\Gamma} \mathbf{p}(\mathbf{x}) \cdot \mathbf{v}(\mathbf{x}) \mathrm{d} A,
\end{aligned}
$$

we arrive then at the sequence of the nonlinear variational equations of the form

$$
\begin{aligned}
& \left(\mathscr{E} \mathbf{v}, \tilde{\mathbf{T}}_{A}\right)=\left\langle\mathbf{v}, f_{A}\right\rangle \forall \mathbf{v} \in V_{0}, \quad \tilde{\mathbf{T}}_{A}(\mathbf{x})=\mathbf{G}_{\delta_{A}(x)}\left(\mathscr{E} \mathbf{w}_{A}(\mathbf{x})\right), \quad \mathbf{x} \in \Omega, \quad \mathbf{w}_{A} \in V_{\mathbf{0}} \\
& (A=1,2, \ldots, M)
\end{aligned}
$$

with the previously calculated $\delta_{A}(\cdot)$. Let us observe that $\delta(\mathbf{x})=1$ implies $(\tilde{\mathbf{T}}(\mathbf{x}) \mathbf{n}(\mathbf{x}))_{T}=\mathbf{0}$ and $(\tilde{\mathbf{T}}(\mathbf{x}) \mathbf{n}(\mathbf{x}))_{N} \leqq \mathbf{0}$. Hence if the condition $\delta(\mathbf{x})=1$ holds in a certain part $\Delta$ of $\Omega$, then the solution to (6.6) may not exist for an arbitrary system of external forces, i. e. the equilibrium equations

$$
\operatorname{div} \widetilde{\mathbf{T}}(\mathbf{x})+\tilde{\varrho} b(\mathbf{x})=\mathbf{0}, \quad \mathbf{x} \in \Omega \cap \Delta ; \quad \tilde{T}(\mathbf{x}) \mathbf{n}_{\partial \Omega}(\mathbf{x})=\mathbf{p}(\mathbf{x}), \quad \mathbf{x} \in \Gamma \cap \Delta,
$$

may not be satisfied for an arbitrary $\mathbf{b}(\mathbf{x})$ and $\mathbf{p}(\mathbf{x})$. It means that the totally delaminated part of a composite (within the continuum model of delamination) is not able to sustain certain systems of loadings. 
Let us introduce the spaces

$$
\begin{aligned}
& V \equiv\left(H^{1}(\Omega)\right)^{\mathbf{3}}, \quad V_{0} \equiv\left\{\mathbf{v} \in V:\left.\mathbf{v}\right|_{\Gamma_{\mathbf{0}}}=\mathbf{0}\right\}, \\
& \Sigma \equiv\left\{\mathbf{T} \in\left(L^{2}(\Omega)\right)^{3 \times 3}: \operatorname{div} \mathbf{T} \in\left(L^{2}(\Omega)\right)^{\mathbf{3}\}},\right.
\end{aligned}
$$

and assume that the delamination density $\delta=\delta(\cdot)$ ensures the coercivness of the operator $\mathbf{G}_{\delta}(\cdot)$ defined on $\left(L^{2}(\Omega)\right)^{3 \times 3}$. Then for every $f \in V_{0}^{* *}$ and every $\Gamma_{0}$ with mes $\left(\Gamma_{0}\right)>0$ there exists the unique solution of $(6.6)$ such that $\mathrm{w}_{A} \in V_{0}, \tilde{\mathrm{T}}_{A} \in \Sigma$. More detailed analysis of this problem will be given elsewhere.

\section{Final remarks}

The proposed mathematical models of delamination processes in laminates, due to their relatively simple form, may constitute the basis not only for the theoretical analysis but also for various engineering applications including the numerical solution of special problems. Using the time local approximation and the pertinent step by step procedure we can calculate, after every step, the delamination density up to the time instant in which the solution does not exist. This procedure makes it also possible to determine the parts of the composite where the total delamination takes place in the deformation process under consideration. The analysis of such situations will be performed separately. It must be emphasized, however, that the models proposed involve four material parameters $\gamma_{N}, \gamma_{T}, \sigma_{0}, \sigma_{1}$ which determine the properties of the bonding between the interfaces of adjacent sheets in laminates. Thus, for the quantitative analysis of special problems, the forementioned parameters have to be previously evaluated on the basis of experimental results combined with the heuristic assumptions introduced in this contribution.

\section{References}

1. Naniewicz, Z.; Woźniak, Cz.: On a quasi-stationary model of debonding processes in layered composites. Ing. Arch. 58 (1988) $403-412$

2. Woźniak, Cz. : A nonstandard method of modelling of thermoelastic periodic composites. Int. J. Eng. Sci. 25 (1987) $483-498$

3. Robinson, A.: Non-standard analysis. Amsterdam: North Holland 1966

4. Woźniak, Cz.: Nonstandard analysis in mechanies. Adv. Mech. 9 (1986) 3-35

Received March 28, 1989

Prof. Dr. Cz. Woźniak

Institute of Fundamental Technological Research

Polish Academy of Science

Swiętokrzyska 21

PL-00-043 Warszawa

Poland 